\title{
COHOMOLOGY OF INDUCED MODULES IN RINGS OF DIFFERENTIAL OPERATORS
}

\author{
by KENNETH A. BROWN and THIERRY LEVASSEUR
}

(Received 17th April 1986)

\section{Introduction}

1.1. Let $K$ be a field of characteristic zero and let $\Delta=\left\{\delta_{1}, \ldots, \delta_{n}\right\}$ be a set of commuting $K$-derivations of the commutative Noetherian $K$-algebra $R$. Let $S=R\left[X_{1}, \ldots, X_{n}\right]$ be the corresponding ring of differential operators, so $\left[X_{i}, r\right]=X_{i} r-r X_{i}=\delta_{i}(r)$, and $\left[X_{i}, X_{j}\right]=0$, for $1 \leqq i, j \leqq n$. Let $M$ be a maximal ideal of $R$ with $R / M$ of finite dimension over $K$. The purpose of this note is to describe the groups

$$
E^{*}:=\left\{\operatorname{Ext}_{S}^{i(S / S M, S / S M): i \geqq 0}\right\}
$$

The dimension $s$ over $R / M$ of the subspace of $\operatorname{Hom}_{R / M}\left(M / M^{2}, R / M\right)$ generated by the image of $\Delta$ is called the differential codimension of $M$ with respect to $\Delta$ [3, Proposition 2.1]. Let $V=\{v \in S: M v \subseteq S M\}$, the idealiser of $S M$, and put $V_{0}=V / S M$; note that the groups $E^{*}$ are $\left(V_{0}-V_{0}\right)$-bimodules.

1.2. The result we shall prove is the following.

Theorem. Let the hypotheses and notation be as in 1.1.

(i) Let $M_{1}$ and $M_{2}$ be distinct maximal ideals of $R$. Then $\operatorname{Ext}_{S}^{*}\left(S / S M_{1}, S / S M_{2}\right)=0$.

(ii) $V_{0}$ is a polynomial algebra in $(n-s)$ variables over $R / M$.

(iii) There is a sequence $\left\{x_{1}, \ldots, x_{s}\right\}$ of elements of $M$, whose images in $R_{M}$ form an

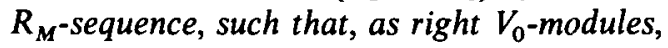

$$
\operatorname{Ext}_{S}^{i}(S / S M, S / S M) \cong \operatorname{Ext}_{\bar{R}}^{i}(R / M, R / M) \otimes_{R} V_{0},
$$

for all $i \geqq 0$, where $\bar{R}=R /\left\langle x_{1}, \ldots, x_{s}\right\rangle$. If $R$ is $M$-adically complete, then $\bar{R}$ is isomorphic to the centraliser in $R$ of $\Delta$.

1.3. There is a special case of the above result which is well-known, (although we have not been able to find an explicit statement of it in the literature). Namely, let $X$ be a non-singular affine variety over an algebraically closed field $K$ characteristic 0 , let $R=\mathcal{O}(X)$ be the ring of regular functions on $X$, and let $M$ be a maximal ideal of $R$. Let $D$ be the ring of differential operators on $X$. Then $\operatorname{Ext}_{D}^{p}(D / D M, D / D M)$ is $K$ for $p=0$, and 0 for $p>0$. For, we may replace $R$ by its $M$-adic completion (as in 3.1); in doing so 
we replace $D$ by $\hat{D}=K\left[\left[X_{1}, \ldots, X_{n}\right]\right]\left[\partial / \partial X_{1}, \ldots, \partial / \partial X_{n}\right][2$, Ch. 3, Lemma 1.5$]$, and the result for $\hat{D}$ can be obtained by a much simplified version of the proof given belowessentially only 2.1 and 3.1 are needed.

1.4. Routine arguments using Shapiro's Lemma [1, page 109] or [5, Theorem 11.65] show that, if $R / M \cong K^{\prime}$, (so $\operatorname{dim}_{K} K^{\prime}=m<\infty$ by hypothesis), we can, in proving 1.1 (ii) and (iii), replace $R$ by $R^{\prime}=K^{\prime} \otimes_{K} R$ and $S$ by $S^{\prime}=K^{\prime} \otimes_{K} S$, so that $K^{\prime} \otimes_{K}(R / M)$ is the direct sum of $m$ copies of $K^{\prime}$. We leave the details of these reductions to the reader. We shall assume throughout Sections 3 and 4 that $R / M=K$.

1.5. The proof of the theorem is organised as follows. A result on injective hulls of simple $R$-modules is obtained in Section 2, and this is used to handle linearly independent derivations in Section 3, first for the easier case where $R$ is complete, and then in general. The main result is proved in Section 4.

\section{The injective hull of the residue field of a local ring}

Lemma 2.1. Let $K$ be a field, let $X_{1}, \ldots, X_{s}$ be commuting indeterminates, and put $A=K\left[\left[X_{1}, \ldots, X_{s}\right]\right]$. Let $D=A\left[Y_{1}, \ldots, Y_{s}\right]$, where $\left[Y_{i}, Y_{j}\right]=0$ and $\left[Y_{i}, a\right]=\partial a / \partial X_{i}$, for $1 \leqq i$, $j \leqq s$ and $a \in A$. Let $M$ be the maximal ideal of $A$. Then $D / D M$ is isomorphic to $E_{A}(K)$ as $A$-modules.

Proof. By [1, p. 173, ex. 32], $E_{A}(K)$ is isomorphic to $K\left[Y_{1}, \ldots, Y_{s}\right]$, where $X_{j} \cdot p(Y)=$ $\partial_{p} / \partial Y_{j}$ for $1 \leqq j \leqq s$ and $p(Y) \in K\left[Y_{1}, \ldots, Y_{s}\right]$. Now $D / D M$ has $K$-basis afforded by the monomials in $\left\{Y_{1}, \ldots, Y_{s}\right\}$; and if $\tau$ is one such monomial, $X_{j}(\tau+D M)=\left(-\partial \tau / \partial Y_{j}+D M\right)$ for $1 \leqq j \leqq s$. The result follows.

Lemma 2.2. Let $R_{1}, R_{2}$ be commutative Noetherian rings containing a subfield $K$. For $i=1,2$, let $M_{i}$ be an ideal of $R_{i}$ with $R_{i} / M_{i} \cong K$, and set $E_{i}=E_{R_{i}}\left(R_{i} / M_{i}\right)$. Let $R=R_{1} \otimes_{K} R_{2}$ and $E=E_{1} \otimes{ }_{K} E_{2}$ (so $E$ is an $R$-module in the obvious way). Then $E=E_{R}\left(R_{1} / M_{1} \otimes R_{2} / M_{2}\right)$.

Proof. Let $G=\operatorname{Hom}_{R_{1}}\left(R_{1} / M_{1},-\right)$, so if $X$ is an $R$-module, $G X=\left\{x \in X: M_{1} x=0\right\}$, and $G$ is a left exact functor from $R$-modules to $R_{2}$-modules. Note that if $X$ is $R$-injective, then $G X$ is $R_{2}$-injective. Let $F=\operatorname{Hom}_{R_{2}}\left(R_{2} / M_{2},-\right)$, a left exact functor from $R_{2}$-modules to abelian groups.

Apply the five term exact sequence of cohomology [5, Theorem 11.2] to obtain

$$
\operatorname{Ext}_{R_{2}}^{1}\left(R_{2} / M_{2}, \operatorname{Hom}_{R_{1}}\left(R_{1} / M_{1}, E\right)\right) \rightarrow \operatorname{Ext}_{R}^{1}(R / I, E) \rightarrow \operatorname{Hom}_{R_{2}}\left(R_{2} / M_{2}, \operatorname{Ext}_{R_{1}}^{1}\left(R_{1} / M_{1}, E\right)\right)
$$

where $I=M_{1} R+M_{2} R$. The two outside groups are zero, and hence so is $\operatorname{Ext}_{R}^{1}(R / I, E)$. Since $E$ is clearly an essential extension of $R / I$, this is sufficient to ensure that $E$ is injective, by the Artin-Rees theorem [6, p. 255, Theorem 4']. 
Corollary 2.3. Continue with the notation of 2.2. Let $0 \rightarrow R_{1} / M_{1} \rightarrow \mathrm{E}_{1}^{*}$ be a minimal injective resolution of $R_{1}$-modules. Then

$$
\left(^{*}\right) 0 \rightarrow R_{1} / M_{1} \otimes_{K} E_{2} \rightarrow \mathrm{E}_{1}^{*} \otimes_{K} E_{2}
$$

is a minimal $R$-injective resolution of $E_{2}$, where $E_{2}$ is viewed as an $R$-module with $M_{1} E_{2}=0$.

Proof. Each term in $E_{1}^{*}$ is a finite direct sum of copies of $E_{1}$, so each term of $\mathbf{E}_{1}^{*} \otimes E_{2}$ is $R$-injective by 2.2 . The sequence $\left(^{*}\right)$ is exact because $K$ is a field, so it is an $R$-injective resolution of $E_{2}$. If $d_{i}: E_{1}^{i} \rightarrow E_{1}^{i+1}$, then $\operatorname{socle}\left(E_{1}^{i+1}\right) \subseteq \operatorname{im} d_{i}$ by hypothesis, so

$$
\operatorname{socle}\left(E_{1}^{i+1} \otimes E_{2}\right)=\operatorname{socle}\left(E_{1}^{i+1}\right) \otimes E_{2} \subseteq \operatorname{im}\left(d_{i} \otimes 1\right)
$$

hence $\left(^{*}\right)$ is a minimal resolution.

\section{Linearly independent derivations}

3.1. Throughout Sections 3 and 4 the notation will be that introduced in 1.1. As noted in 1.4 , we shall assume that $R / M=K$. Moreover, let $\hat{R}$ denote the $M$-adic completion of $R$. The derivations $\left\{\delta_{i}\right\}$ extend to a set of commuting derivations of $\hat{R}$, which we denote by the same notation. We can thus form the ring of differential operators $\hat{R}[\mathbf{X} ; \Delta]:=\hat{S}$.

Let $V$ [resp. $\hat{V}]$ be the idealiser of $S M$ in $S$ [resp. of $\hat{S} M$ in $\hat{S}$ ], and let $V_{0}=V / S M$ [resp. $\left.\hat{V}_{0}=\hat{V} / \hat{S} M\right]$ ]. Using the fact that each element of $S / S M$ is killed by a power of $M$, it is easy to check that $\widehat{V}=V+\widehat{S} M$, so that, as rings, $V_{0} \cong \hat{V}_{0}$.

Lemma. Let the notation be as in 1.1 and above.

(i) Let $M_{1}$ and $M_{2}$ be distinct maximal ideals of $R$. Then $\operatorname{Ext}_{S}^{i}\left(S / S M_{1}, S / S M_{2}\right)=0$.

(ii) As left $R$ - and right $V_{0}$-modules,

$$
\begin{aligned}
\operatorname{Ext}_{S}^{*}(S / S M, S / S M) & \cong \operatorname{Ext}_{R}^{*}(R / M, S / S M) \\
& \cong \operatorname{Ext}_{\hat{R}}^{*}(\hat{R} / \hat{R} M, \hat{S} / \hat{S} M) \\
& \cong \operatorname{Ext}_{S}^{*}(\hat{S} / \hat{S} M, \hat{S} / \hat{S} M)
\end{aligned}
$$

Similar identifications can be made with $S_{M}:=R_{M}[X ; \Delta]$ in place of $\hat{S}$.

Proof. By [1, p. 109] or [5, Theorem 11.65].

$$
\operatorname{Ext}_{S}^{i}\left(S / S M_{1}, S / S M_{2}\right) \cong \operatorname{Ext}_{R}^{i}\left(R / M_{1, R} \mid S / S M_{2}\right)
$$


Each element of the right hand module is annihilated by $M_{1}$ and by some power of $M_{2}$, so (i) follows at once. The above isomorphism also yields the first and third isomorphisms of (ii). Since

$$
\begin{aligned}
\operatorname{Ext}_{R}^{*}(\hat{R} / \hat{R} M, \hat{S} / \hat{S} M) & \cong \hat{R} \otimes_{R} \operatorname{Ext}_{R}^{*}(R / M, \hat{S} / \hat{S} M) \\
& \cong \operatorname{Ext}_{R}^{*}(R / M, S / S M)
\end{aligned}
$$

by [5, Theorem 11.65] for the first isomorphism and the comments above the statement of the lemma for the second, we also obtain the second isomorphism of (ii).

3.2. Each $\delta_{i} \in D$ induces an element $\delta_{i}^{*}$ of $\operatorname{Hom}_{R}\left(M / M^{2}, R / M\right)$. Let $s=$ $\operatorname{dim}_{K} \operatorname{span}\left\{\delta_{i}^{*}: 1 \leqq i \leqq n\right\}$; this is the differential codimension of $M$ with respect to $\Delta$ [3, Proposition 2.1]. Renumber $\Delta$ so that $\delta_{1}^{*}, \ldots, \delta_{s}^{*}$ are linearly independent, and put $T=R\left[X_{1}, \ldots, X_{s}\right]$. Choose $x_{1}, \ldots, x_{s} \in M$ with images in $M / M^{2}$ forming part of a dual basis to $\delta_{1}^{*}, \ldots, \delta_{s}^{*}$. Put

$$
I=\left\{r \in R: c r \in\left\langle x_{1}, \ldots, x_{s}\right\rangle, \text { for some } c \in R \backslash M\right\} \text {, and } \bar{R}=R / I \text {. }
$$

Proposition. $\operatorname{Ext}_{T}^{*}(T / T M, T / T M) \cong \operatorname{Ext}_{\tilde{R}}^{*}(R / M, R / M)$.

Proof. Assume first that $R$ is complete. Put $R_{1}=\left\{r \in R: \delta_{i}(r)=0,1 \leqq i \leqq s\right\}$. Since char $K=0, R=R_{1}\left[\left[x_{1}, \ldots, x_{s}\right]\right]$ by [4, Section 4, Theorem 2 and remark at end of section]. Being an image of $R, R_{1}$ is a complete local ring with maximal ideal $M_{1}$, say. Put $Q=M_{1} R$.

Thus $T Q=Q T$ is an ideal of $T$ and $R=R_{1} \otimes_{K} R_{2}$ where $R_{2}=R / Q=K\left[\left[x_{1}, \ldots, x_{s}\right]\right]$, so that $T / T Q=K\left[\left[x_{1}, \ldots, x_{s}\right]\right]\left[X_{1}, \ldots, X_{s}\right]$. Let $M_{2}$ be the ideal $\left\langle x_{1}, \ldots, x_{s}\right\rangle$ of $R_{2}$. By 2.1, $T T M$ is the $K\left[\left[x_{1}, \ldots, x_{s}\right]\right]$-injective hull of $K=R_{2} / M_{2}$. Therefore, in the notation of 2.3 ,

$$
\begin{array}{rlr}
\operatorname{Ext}_{T}^{*}(T / T M, T / T M) & =\operatorname{Ext}_{R}^{*}(R / M, T / T M), & \text { by }[5,11.65], \\
& =\operatorname{socle}\left(\mathbf{E}_{1}^{*} \otimes_{K} T / T M\right), \quad \text { by } 2.3 \\
& =\operatorname{socle}\left(\mathbf{E}_{1}^{*} \otimes_{K} K\right) \\
& =\operatorname{Ext}_{R_{1}}^{*}\left(R_{1} / M_{1}, R_{1} / M_{1}\right)
\end{array}
$$

Now drop the hypothesis that $R$ is complete. The elements $x_{1}, \ldots, x_{s}$ of $\hat{R}$ chosen above can be taken in $R$, so that $R\left\langle x_{1}, \ldots, x_{s}\right\rangle \cap R=I[6, \mathrm{p}$. 257, Corollary 2]. Thus the subring $R_{1}$ of $\hat{R}$ defined above is just the $M /\left\langle x_{1}, \ldots, x_{s}\right\rangle$-adic completion of $\bar{R}[6$, p. 258 Corollary 2], and so

$$
\operatorname{Ext}_{R_{1}}^{*}\left(R_{1} / M_{1}, R_{1} / M_{1}\right)=\operatorname{Ext}_{\bar{R}}^{*}(R / M, R / M) .
$$

The result follows from (1), (2) and 3.1. 


\section{The main result}

4.1. We retain the next three paragraphs the notations of $1.1,3.1$ and 3.2 . For $s+1 \leqq i \leqq n, 1 \leqq j \leqq s$ there exist elements $r_{i j}$ of $K$ such that

$$
\delta_{i}^{*}=\sum_{j=1}^{s} r_{i j} \delta_{j}^{*}
$$

Set $Y_{i}=X_{i}-\sum_{j=1}^{s} r_{i j} X_{j} \in S$, for $s+1 \leqq i \leqq n$. Since

$$
\left(\delta_{i}-\sum_{j=1}^{s} r_{i j} \delta_{j}\right)(M) \subseteq M
$$

for $i \geqq s+1$,

$$
\left[Y_{i}, M\right] \subseteq M
$$

It follows that the subring $U=\left\langle S M, Y_{s+1}, \ldots, Y_{n}\right\rangle$ of $S$ is contained in the idealiser $V$ of $S M$. Since the monomials $\left\{X^{I}: I=\left(i_{1}, \ldots, i_{s}\right) \in \mathbb{N}^{s}\right\}$ form a free right generating set for $S / S M$ as a right $U / S M$-module, it follows easily that

$$
V=\left\langle S M, Y_{s+1}, \ldots, Y_{n}\right\rangle
$$

so that

$$
V_{0}:=V / S M=K\left[Y_{s+1}, \ldots, Y_{n}\right]
$$

is a polynomial algebra over $K$ (proving (ii) of the theorem).

Lemma 4.2. (i) With the notation of 4.1,

$$
S / S M \cong T / T M \otimes_{K} V_{0}=T / T M \otimes_{R} V_{0}
$$

as $\left(T-V_{0}\right)$-bimodules.

(ii) Let $W$ be a finitely generated left $T$-module. Then, as right $V_{0}$-modules,

$$
\operatorname{Ext}_{T}^{*}(W, T / T M) \otimes_{R} V_{0} \cong \operatorname{Ext}_{S}^{*}\left(S \otimes_{T} W, S / S M\right)
$$

Proof. (i) It is easily checked that the map $\psi: S / S M \rightarrow T / T M \otimes_{K} V_{0}$ given by $\psi\left(\left(X^{I}+S M\right) v\right)=\left(X^{I}+T M\right) \otimes v,\left(v \in V_{0}\right)$, is a well-defined bimodule isomorphism. The second equality holds since $T / T M$ and $V_{0}$ are annihilated by $M$.

(ii) Let $C$ be a left $T$-module. Define a map

$$
\Theta: \operatorname{Hom}_{T}(C, T / T M) \otimes_{R} V_{0} \rightarrow \operatorname{Hom}_{S}\left(S \otimes_{T} C, S / S M\right)
$$


by setting, for $s \in S, v \in V_{0}, c \in C$ and $f \in \operatorname{Hom}_{T}(C, T / T M)$,

$$
\Theta(f \otimes v)(s \otimes c)=s \psi^{-1}(f(c) \otimes v)
$$

Routine checks confirm that $\operatorname{im} \Theta$ consists of $S$-homomorphisms, and that $\Theta$ is a homomorphism of right $V_{0}$-modules.

We note next that

$$
\text { when } C=T^{(n)} \text { is free, } \Theta \text { is an isomorphism. }
$$

For in this case, as right $V_{0}$-modules,

$$
\operatorname{Hom}_{T}(C, T / T M) \otimes_{R} V_{0} \cong\left(T / T M \otimes_{R} V_{0}\right)^{(n)},
$$

and

$$
\operatorname{Hom}_{S}\left(S \otimes_{T} C, S / S M\right) \cong(S / S M)^{(n)},
$$

and one sees that $\Theta$ is just the sum of $n$ copies of the isomorphism of (i).

The proof now continues along familiar lines. Let $W$ be a finitely generated left $T$ module, and let

$$
\mathbf{F}_{*} \rightarrow W \rightarrow 0
$$

be a resolution of $W$ by finitely generated free $T$-modules. Apply $\mathrm{Hom}_{T}$ $(-, T / T M) \otimes_{K} V_{0}$ to (7); as $K$ is a field the resulting complex has cohomology

$$
\operatorname{Ext}_{T}^{*}(W, T / T M) \otimes_{K} V_{0}
$$

On the other hand, if we apply $\operatorname{Hom}_{S}\left(S \otimes_{T}-, S / S M\right)$ to (7), then since $S$ is a free $T$ module we get the complex $\operatorname{Hom}_{S}\left(S \otimes \mathrm{F}_{*}, S / S M\right)$, with cohomology

$$
\operatorname{Ext}_{S}^{*}\left(S \otimes_{T} W, S / S M\right)
$$

Notice that the groups (8) can be denoted

$$
\operatorname{Ext}_{T}^{*}(W, T / T M) \otimes_{R} V_{0}
$$

because $\operatorname{Hom}_{T}\left(\mathbf{F}_{*}, T / T M\right) M=\operatorname{Ext}_{T}^{*}(W, T / T M) M=M V_{0}=0$. The desired isomorphism now follows from (9), (10) and the isomorphism

$$
\operatorname{Hom}_{T}\left(\mathbf{F}_{*}, T / T M\right) \otimes_{R} V_{0} \cong \operatorname{Hom}_{S}\left(S \otimes_{T} \mathbf{F}_{*}, S / S M\right)
$$

of right $V$-modules given by $\Theta$. 
4.3. Proof of Theorem 1.2. Parts (i) and (ii) have already been proved in 3.1(i) and 4.1. By Lemma 4.2(ii) and Proposition 3.2,

$$
\begin{aligned}
\operatorname{Ext}_{S}^{*}(S / S M, S / S M) & \cong \operatorname{Ext}_{T}^{*}(T / T M, T / T M) \otimes_{R} V_{0} \\
& \cong \operatorname{Ext}_{R / I}^{*}(R / M, R / M) \otimes_{R} V_{0}
\end{aligned}
$$

where $I=\left\{\tau \in R: c \tau \in\left\langle x_{1}, \ldots, x_{s}\right\rangle, c \in R \backslash M\right\}$, and these are isomorphisms of right $V_{0^{-}}$ modules. Moreover, both $\operatorname{Ext}_{R / I}^{*}(R / M, R / M)$ and $\operatorname{Ext}_{\bar{R}}^{*}(R / M, R / M)$ are isomorphic to $\operatorname{Ext}_{\bar{R}_{M}}^{*}\left(R_{M} / M_{M}, R_{M} / M_{M}\right)$, so (iii) follows.

Acknowledgement. Most of this work was carried out while the second author was visiting the University of Glasgow in July 1985, with financial support from the U.K. S.E.R.C. and the Centenary Fund of the Edinburgh Mathematical Society.

\section{REFERENCES}

1. N. Bourbakı, Algèbre Commutative, Chapter X (Masson, Paris, 1983).

2. J.-E. BJork, Rings of Differential Operators (North-Holland, Amsterdam, 1979).

3. K. GoodearL and T. H. Lenegan, Krull dimension of differential operator rings IV-multiple derivations, Proc. London Math. Soc. (3) 47 (1983), 306-336.

4. J. Lipman, Free derivation modules on algebraic varieties, Amer. J. Math. 87 (1965), 874-898.

5. J. Rotman, An Introduction to Homological Algebra (Academic Press, New York, 1979).

6. O. Zariskı and P. SAmuel, Commutative Algebra, Volume II (Van Nostrand, Princeton, 1960).

Department of Mathematics

UNIVERSITY OF GLASGOW

GLASGOW G11 5HF

SCOTLAND
Departement de Mathématioues

Universite de Paris VI

TOUR 45-46-5

4 PLACE JUSSIEU

Paris

France 